\title{
Atomic packing and short-to-medium range order evolution of Zr-Pd metallic glass
}

\author{
QI Li ${ }^{*}$, LIU Mei, ZHANG ShiLiang, ZHANG XinYu \& TAN ChunLin \\ State Key Laboratory of Metastable Materials Science \& Technology, Yanshan University, Qinhuangdao 066004, China
}

Received June 1, 2011; accepted August 31, 2011

\begin{abstract}
A larger-scale $\mathrm{Zr}_{70} \mathrm{Pd}_{30}$ alloy system has been simulated using molecular dynamics (MD) to investigate structure evolution in $\mathrm{Zr}_{70} \mathrm{Pd}_{30}$ metallic glass. The simulated pair distribution function of $\mathrm{Zr}_{70} \mathrm{Pd}_{30}$ metallic glass agrees well with the experimental results. Voronoi polyhedron analysis indicates that the icosahedra are not randomly distributed in space, but form characteristic intercrossed icosahedral clusters with medium-range order. Intercrossed icosahedral clusters are the dominant local configurations in $\mathrm{Zr}_{70} \mathrm{Pd}_{30}$ metallic glass and probably cause the quasicrystalline phase discovered in $\mathrm{Zr}_{70} \mathrm{Pd}_{30}$ metallic glass.
\end{abstract}

molecular dynamics simulation, $\mathbf{Z r}_{70} \mathbf{P d}_{30}$ metallic glass, icosahedra, medium-range order

Citation: $\quad$ Qi L, Liu M, Zhang S L, et al. Atomic packing and short-to-medium range order evolution of Zr-Pd metallic glass. Chinese Sci Bull, 2011, 56: 39083911, doi: 10.1007/s11434-011-4841-0

In recent years, quasicrystals have been found to form in many $\mathrm{Zr}$ based binary metallic glasses (MGs) such as $\mathrm{Zr}-\mathrm{Pd}$ and $\mathrm{Zr}$-Pt [1,2]. The bulk quasicrystalline alloys exhibit high strength and good ductility [3]. In the Zr-Pd binary system, the nature of the amorphous-to-quasicrystalline phase transition has been clarified [4,5]. However, the characteristic structure in the $\mathrm{Zr}_{70} \mathrm{Pd}_{30}$ MGs is currently unknown. We also do not know whether the structures of $\mathrm{Zr}_{70} \mathrm{Pd}_{30}$ MGs directly affect the transition into the quasicrystalline phase. In the present study, the transition of the $\mathrm{Zr}_{70} \mathrm{Pd}_{30}$ alloy from the supercooled liquid to the solid is simulated using molecular-dynamics (MD). The structure of the $\mathrm{Zr}_{70} \mathrm{Pd}_{30}$ metallic glass is investigated in detail by means of the pair distribution function (PDF), pair analysis method (PA) [6,7] and Voronoi polyhedron analysis method.

Based on the hypothesis that linking icosahedra can form the quasicrystalline phase, there has been considerable interest in the icosahedral medium range ordering (IMRO) of MGs's [8-13], which probably affects the glass formability as well as the mechanical properties, including Young's modulus and shear banding resistance [14]. Recently, some

*Corresponding author (email: qili@ysu.edu.cn) models for IMRO in MGs have been suggested by experimental and computational studies [15-22].

\section{Simulation methods}

Theoretical models of liquid and amorphous structures are often obtained from MD simulations using either $a b$ initio methods or empirical pair potentials. However, finite computational resources restrict the size of the ensembles that can be considered in ab initio calculations to a few hundred atoms. The systems simulated with pair potentials show an increase in the number of icosahedra during liquid cooling [23-26]. Therefore, the embedded-atom method (EAM) potential [27], which has been successfully applied to a wide range of aspects of the liquid and amorphous states, is adopted in the present simulations [28-33].

Based on the constant temperature and constant pressure (NPT) method, the simulations are performed with a system consisting of 100000 atoms in a cubic box with periodic boundary conditions along all three directions. The Newton equations were integrated using the Verlet [34] algorithm with a time step of $5 \mathrm{fs}$. The initial liquid state was obtained 
by holding the system well above a liquidus temperature. The system was melted, homogenized, and then quenched by rescaling particle velocities to room temperature at a rate of $1 \times 10^{12} \mathrm{~K} / \mathrm{s}$. The dependences of the volume and the local structure on the temperature were examined. The structural analysis of liquids and glasses was performed using the PA technique and index of Honeycutt-Anderson in a separate program. Using the PA technique, the fivefold symmetry bonds plus the icosahedral short-range ordering can be easily seen at the atomic level. For the larger clusters with IMRO, the local structure in the models was analyzed using the Voronoi method.

\section{Results and discussions}

Since the PDF is a Fourier transformation of the structure factor obtained from a diffraction experiment, it can be used to compare the simulation results with the experimental ones for liquid and glass structures. Figure 1 shows the evolution of the total PDF during the rapid quenching process of $\mathrm{Zr}_{70} \mathrm{Pd}_{30}$ liquid alloy at a cooling rate of $1 \times 10^{12} \mathrm{~K} / \mathrm{s}$. The splitting of the second peak in the total PDF becomes pronounced with decreasing temperature, which indicates the formation of $\mathrm{Zr}_{70} \mathrm{Pd}_{30}$ metallic glass and the enhancement of ISRO during the quenching process. The simulated total PDFs for the liquid and glass structures both agree well with the experimental results, although their first peaks are slightly higher.

The glass transition temperature $T_{\mathrm{g}}$ can be obtained from the relationship between the Wendt-Abraham ratio $R$ [35] and temperature. In Figure 2, both the proportion of icosahedra and $R$ are represented as functions of temperature for a cooling rate of $1 \times 10^{12} \mathrm{~K} / \mathrm{s}$. The glass transition temperature $T_{\mathrm{g}}$ is about $700 \mathrm{~K}$, which is lower than the experimental value $(\approx 750 \mathrm{~K})$ [18]. Considering that the much higher cooling rate adopted in the present simulation compared with that occurring experimentally will reduce the $T_{\mathrm{g}}$, our

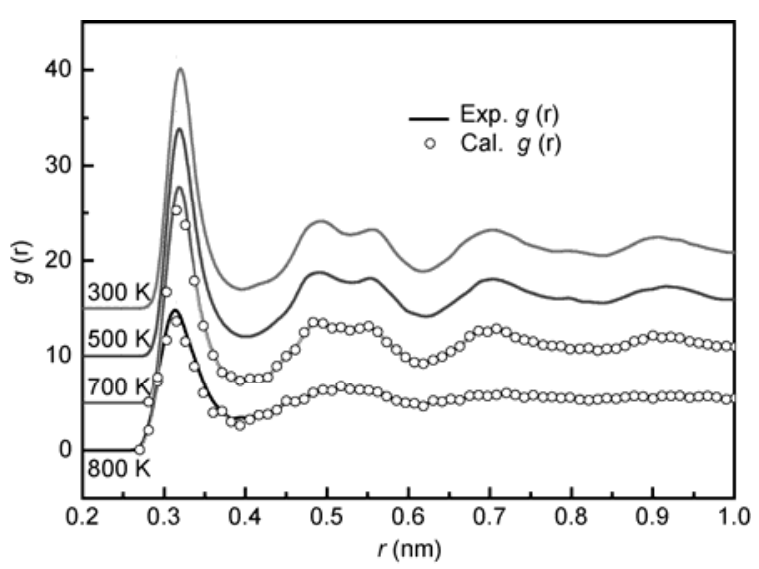

Figure 1 Evolution of total PDF during the liquid quenching process of $\mathrm{Zr}_{70} \mathrm{Pd}_{30}$ alloy.

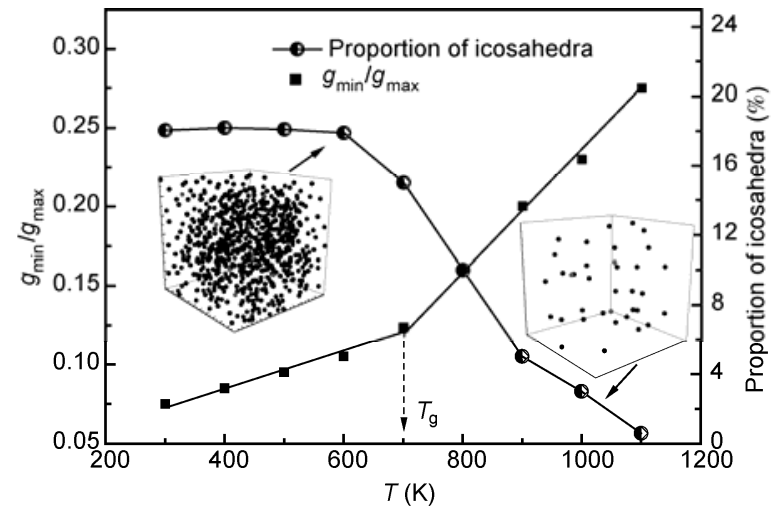

Figure 2 Relationship between Wendt-Abraham ratio $R=g_{\min } / g_{\max }$ and temperature obtained from Figure 1, and the proportion of icosahedra during the quenching process from the liquid state at a cooling rate of $1 \times 10^{12}$ $\mathrm{K} / \mathrm{s}$. The two embedded images are spatial distributions of the center atoms of icosahedra at 1100 and $600 \mathrm{~K}$, respectively.

calculations are reasonable. There is a decrease in the proportion of icosahedra going from slightly above $T_{\mathrm{g}}$ to $T_{\mathrm{g}}$; this fact has already been reported in many MD studies $[10,36]$.

The PDF reflects the statistical average of the ISRO structures, but is incapable of describing the topological structures of the ISRO. The pair analysis method has been widely used to assess the local configurations of the liquid, amorphous and crystal structures. In this method, a set of four integers, $i j k l$, is designed to describe the different local configurations. The first integer $i$ is used to identify the bonding of two given atoms. $i$ is 1 when they are bonded in the root pair, otherwise $i$ is 2 . The second integer $j$ is the number of common near-neighbor atoms shared by the root pair. The third integer $k$ is the number of bonds among the shared neighbors. The fourth integer $l$ is needed to distinguish configurations having the same first three indices but being different bond geometries. Different local atomic structures have different PA indices and general observations are as following: the fcc crystal structure leads only to 1421 bonds; the hcp crystal structure leads to 50\% 1421 bonds and $50 \% 1422$ bonds; and the bcc crystal structure has $43 \% 1441$ bonds and $57 \% 1661$ bonds. The 1551 bond represents the two neighboring atoms with five common neighbors that form a pentagon of near-neighbor contacts, and is therefore situated in a fivefold symmetry environment. The number of 1551 bonds is a direct measurement of the degree of icosahedral ordering. The 13-atom icosahedron has twelve 1551 bond-types. When one bond between a pair of outer atoms in an icosahedron is broken, two of the 1551 bonds become transformed into 1541 bonds and two are transformed into 1431 bonds.

Figure 3 shows the evolution of the relative numbers of various bonds during the rapid quenching process of $\mathrm{Zr}_{70} \mathrm{Pd}_{30}$ alloy. It is clear that the numbers of 1551,1541 and 1431 bonds which are closely related to icosahedra or defective icosahedra, are significantly higher than the other 
types; their summation reaches $74.2 \%$ of the glass structure at $300 \mathrm{~K}$. Moreover, the number of 1551 bonds increases remarkably during the glass transition process, whereas others only change a little. This means that various icosahedral ISRO structures in the $\mathrm{Zr}_{70} \mathrm{Pd}_{30}$ alloy are inherent to the glass structure during the rapid quenching process, and the icosahedral ISRO structures play a critical role in the formation of the $\mathrm{Zr}_{70} \mathrm{Pd}_{30}$ metallic glass. Although the existence of ISRO in the glassy state of some metals has been proven, in some MGs the ISRO is absent. The first evidence for structural identification of the icosahedral cluster in the $\mathrm{Zr}_{70} \mathrm{Pd}_{30}$ glassy alloy was provided by Saida et al. [18], which presents a high-resolution TEM image of the $\mathrm{Zr}_{70} \mathrm{Pd}_{30}$ glassy alloy. Although the diffraction spots are quite weak due to the small area of the ordered region and the fine electron beam, the fivefold symmetry is clearly identified, which indicates that the cluster has an icosahedral structure. These results imply that the icosahedral clusters observed in the as-quenched state are attributable to the high stability of the icosahedral atomic configuration during the cooling process from the melt [18].

One can now ask what kinds of relationships are there among the large number of icosahedral or defective icosahedral clusters, and also what further kinds of characteristic IMRO structure the $\mathrm{Zr}_{70} \mathrm{Pd}_{30}$ glassy alloy has. To answer these questions, we adopt the Voronoi polyhedron analysis method. Voronoi polyhedron analysis gives hundreds of types of polyhedra, among which the most popular polyhedron corresponds to the icosahedral atomic configuration. Just as the 1551 bond only reveals the presence of fragments of icosahedra, the PA method is incapable of characterizing the larger clusters with IMRO. To examine the local structure in the fully relaxed glass models, the atomic coordinates in the models were analyzed using the Voronoi method. Based on the pattern of two linking icosahedra, there are four types of configuration: vertex-sharing (VS), edge-sharing (ES), face-sharing (FS) and intercross-sharing

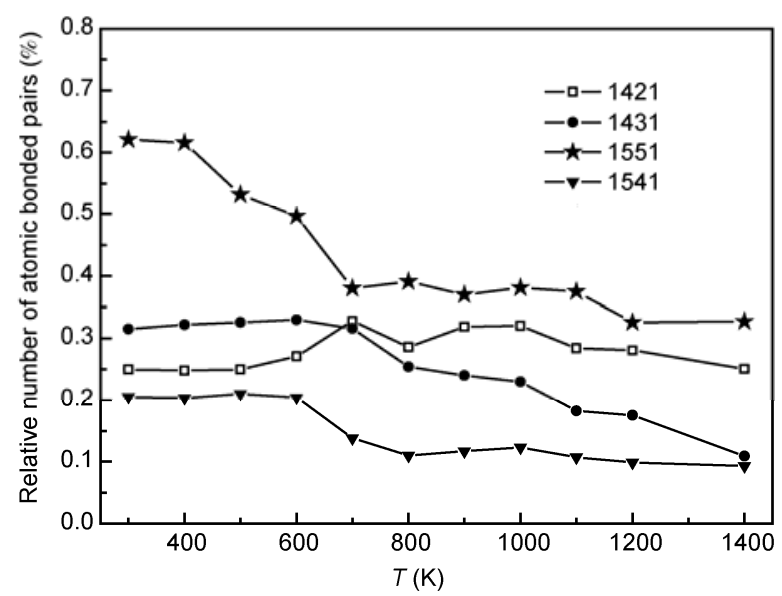

Figure 3 Evolution of relative numbers of different bond types during the rapid quenching process.
(IS) atoms, as shown in the four embedded images in Figure 4. Thus, IMRO structure with extended icosahedral clusters can be formed. Figure 4 also shows the evolutions of the four types of configuration with temperature during rapid quenching. The figure shows that at high temperature, the numbers of VS, ES, FS and IS clusters are small, indicating that most icosahedra in the system are isolated from each other. As the temperature continuously decreases, more and more icosahedra are linked with each other to form VS, ES, FS and IS types. Among the four cluster bond-types, the IS bond-type increases remarkably and is the dominant cluster bond-type in the supercooled liquid and glass structures. This indicates that the extended icosahedral clusters in the $\mathrm{Zr}_{70} \mathrm{Pd}_{30}$ supercooled liquid and glass structures are mainly composed of the IS cluster bond-type. Since the central atoms of two icosahedra bond with each other by sharing four atoms in the IS cluster bond-type as shown in Figure 4, it is high densely packed and will be more stable than other cluster bond-types. Based on the idea that linking icosahedra can form quasicrystals, we can infer that the intercrossed icosahedral clustering with medium-range order is the direct reason for the formation of the quasicrystalline phase discovered in the $\mathrm{Zr}_{70} \mathrm{Pd}_{30}$ metallic glass.

\section{Conclusion}

The present study investigated the atomic structure and the relationship between the IMRO and quasicrystal transition of $\mathrm{Zr}_{70} \mathrm{Pd}_{30}$ alloy using the binary amorphous MD model. The simulation results show that highly icosahedral clusters exist in the amorphous state. The detailed analysis of the cooling process from liquid showed that the icosahedral structure does not form randomly. More intercrossing icosahedral clusters form, leading to the characteristic network structures in the glassy state. This is likely to be the direct reason for the quasicrystalline phase discovered in the $\mathrm{Zr}_{70} \mathrm{Pd}_{30}$ metallic glass.

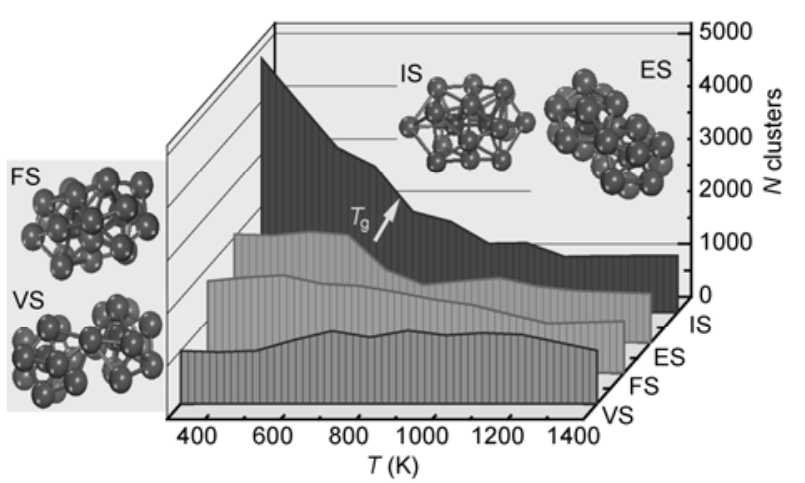

Figure 4 Evolutions of the numbers of VS, ES, FS, and IS clusters in the system with temperature at a cooling rate of $1 \times 10^{12} \mathrm{~K} / \mathrm{s}$. The four embedded images are schematic configurations of the VS, ES, FS, and IS clusters between two icosahedra. 
This work was supported by the National Basic Research Program of China (2010CB731600) and the National Natural Science Foundation of China (50901066, 50771090 and 50821001).

1 Saida J, Matsushita M, Inoue A. Nanoscale icosahedral quasicrystalline phase formation in a rapidly solidified. Appl Phys Lett, 2000, 77: 73-77

2 Murty B S, Ping H D, Hono K. Nanoquasicrystallization of binary Zr-Pd metallic glasses. Appl Phys Lett, 2000, 77: 1102-1106

3 Inoue A, Zhang T, Chen M W, et al. Ductile quasicrystalline alloys. Appl Phys Lett, 2000, 76: 967-972

4 Jiang J Z, Zhuang Y X, Rasmussen H, et al. Formation of quasicrystals and amorphous-to-quasicrystalline phase transformation kinetics in $\mathrm{Zr}_{65} \mathrm{Al}_{7.5} \mathrm{Ni}_{10} \mathrm{Cu}_{7.5} \mathrm{Ag}_{10}$ metallic glass under pressure. Phys Rev $\mathrm{B}$, 2001, 64: 094208

5 Jiang J Z, Saksl K, Saida J, et al. Evidence of polymorphous amorphous-to-quasicrystalline phase transformation in $\mathrm{Zr}_{66.7} \mathrm{Pd}_{33.3}$ metallic glass. Appl Phys Lett, 2002, 80: 781-783

6 Honeycut J D, Andersen H C. Molecular dynamics study of melting and freezing of small Lennard-Jones clusters. J Phys Chem, 1987, 91: 4950-4954

7 Qi L, Li M M, Ma M Z, et al. High pressure effect on structural transition of Fe cluster during rapid quenching processes. Sci China Phys Mech Astron, 2010, 53: 2037-2041

8 Schenk T, Holland-Moritz D, Simonet V, et al. Icosahedral shortrange order in deeply undercooled metallic melts. Phys Rev Lett, 2002, 89: 075507

9 Park J, Shibutani Y. Effects of atomic size for Voronoi tessellation technique on binary and ternary systems of metallic glasses. Mater Trans, 2006, 47: 2904-2909

10 Jónsson H, Andersen H C. Icosahedral ordering in the Lennard-Jones liquid and glass. Phys Rev Lett, 1988, 60: 2295-2298

11 Wang Q, Wang L M, Ma M Z, et al. Diffusion-controlled crystal growth in deeply undercooled $\mathrm{Zr}_{50} \mathrm{Cu}_{50}$ melt on approaching the glass transition. Phys Rev B, 2011, 83: 014202

12 Hui X D, Liu X J, Gao R, et al. Atomic structures of Zr-based metallic glasses. Sci China Ser G-Phys Mech Astron, 2008, 4: 400-413

13 Li G, Liu R P, Li Y C, et al. Stable structure of $\mathrm{Zr}_{49} \mathrm{Cu}_{44} \mathrm{~A}_{17}$ metallic glass matrix composite with $\mathrm{CuZr}$ phase under high pressure up to 40.8 GPa. Chinese Sci Bull, 2011, 56: 372-375

14 Peng H L, Li M Z, Wang W H. Structural signature of plastic deformation in metallic glasses. Phys Rev Lett, 2011, 106: 135503

15 Hirata A, Guan P F, Fujita T, et al. Direct observation of local atomic order in a metallic glass. Nat Mater, 2011, 10: 28-33

16 Cheng Y Q, Ma E, Sheng H W. Atomic level structure in multicomponent bulk metallic glass. Phys Rev Lett, 2009, 102: 245501

17 Kuo W K, Sheng H W, Alamgir F M, et al. Icosahedral short-range order in amorphous alloys. Phys Rev Lett, 2004, 92: 145502
18 Saida J, Matsushita M, Inoue A. Direct observation of icosahedral cluster in $\mathrm{Zr}_{70} \mathrm{Pd}_{30}$ binary glassy alloy. Appl Phy Lett, 2001, 79: 412-414

19 Jing Q, Zhang B, Zhang J, et al. Electrochemical corrosion and oxidation resistances of $\mathrm{Zr}_{60} \mathrm{Ni}_{21} \mathrm{Al}_{19}$ bulk amorphous alloys. Sci China Phys Mech Astron, 2010, 53: 2223-2226

20 Ma M Z, Zong H T, Wang H Y, et al. The fluidity and molding ability of glass-forming Zr-based alloy melt. Sci China Ser G-Phys Mech Astron, 2008, 51: 438-444

21 Qi L, Dong L F, Zhang S L, et al. Glass formation and local structure evolution in rapidly cooled $\mathrm{Pd}_{55} \mathrm{Ni}_{45}$ alloy melt: Molecular dynamics simulation. Comput Mater Sci, 2008, 42: 713-716

22 Zong H T, Ma M Z, Wang L M, et al. Effects of minor addition on glass forming ability: Thermal versus elastic criteria. J Appl Phys, 2010, 107: 053515

23 Jing Q, Xu Y, Zhang X Y, et al. Zr-Cu amorphous films prepared by magnetron co-sputtering deposition of pure $\mathrm{Zr}$ and $\mathrm{Cu}$. Chin Phys Lett, 2009, 26: 086109

24 Zhang B Q, Zhang X Y, Li G, et al. Effect of yttrium addition on glass forming ability of ZrCuAlSi alloy. Chin Phys Lett, 2009, 26: 066105

25 Li G, Gao Y P, Sun Y N, et al. Undercooling and solidification of $\mathrm{Ni}_{77} \mathrm{P}_{23}$ alloy in a 52-m drop tube. Chin Phys B, 2008, 17: 3412-3415

26 Yonezawa F. Solid State Physics. London: Academic Press, 1991. 179-254

27 Daw M S, Baskes M I. Model of metallic cohesion: The embedded-atom method. Phys Rev B, 1984, 29: 6443-6447

28 Li G, Liu J H, Wang W K, et al. Influence of pressure on the solid phase transformation of Cu-Al-Bi alloy. Chin Phys B, 2010, 19: 096202

29 Zong H T, Ma M Z, Liu L, et al. $\mathrm{W}_{\mathrm{f}} / \mathrm{Zr}_{41.2} \mathrm{Ti}_{13.8} \mathrm{Cu}_{12.5} \mathrm{Ni}_{10} \mathrm{Be}_{22.5}$ bulk metallic glass composites prepared by a new melt infiltrating method. J Alloy Compd, 2010, 504: 106-109

30 Li G, Li Y C, Jing Q, et al. Atomic configuration evaluation of $\mathrm{Zr}-\mathrm{Ni}$-Al bulk metallic glass under high pressure. Mater Chem Phys, 2009, 113: 937-940

31 Kondo T, Tsumuraya K. Isosahedral clustering in a supercooled liquid and glass. J Chem Phys, 1991, 94: 8220

32 Rapaport D C. The Art of Molecular Dynamics Simulation. New York: Cambridge University Press, 2004. 332-343

33 Jing Q, Zhao L, Yuan H, et al. Crystallization and thermal fatigue of ZrTiCuNiBe bulk amorphous alloys heated by laser pulses. Sci China Phys Mech Astron, 2010, 53: 419-423

34 Verlet L. Computer "experiments" on classical fluids. I. Thermodynamical properties of Lennard-Jones molecules. Phys Rev, 1967, 159: 98-102

35 Abraham F F. Functional dependence of drag coefficient of a sphere on Reynolds number. Phys Rew E, 1978, 41: 1244-1248

36 Wang L, Bian X, Zhang J. Structural simulation of clusters in liquid $\mathrm{Ni}_{50} \mathrm{Al}_{50}$ alloys. Model Simul Mater Sci Eng, 2002, 10: 331-335

Open Access This article is distributed under the terms of the Creative Commons Attribution License which permits any use, distribution, and reproduction in any medium, provided the original author(s) and source are credited. 